\title{
A New Hybrid PSS Optimization Method Based on Improved Active Set Algorithm
}

\author{
Xiang-Yu Liu, ${ }^{1}$ Yu-Ling He $\mathbb{D}^{2},{ }^{2}$ and Jian $\mathrm{Yao}^{3}$ \\ ${ }^{1}$ State Grid Hebei Electric Power Research Institute, Shijiazhuang 050021, China \\ ${ }^{2}$ Institute of Equipment Fault Diagnosis and Testing Technology, North China Electric Power University, Baoding 071003, China \\ ${ }^{3}$ Jiangsu Lianneng Electric Power Research Institute, Nanjing 210018, China \\ Correspondence should be addressed to Yu-Ling He; heyuling1@163.com
}

Received 28 July 2017; Revised 26 January 2018; Accepted 26 February 2018; Published 3 April 2018

Academic Editor: Ton D. Do

Copyright (C) 2018 Xiang-Yu Liu et al. This is an open access article distributed under the Creative Commons Attribution License, which permits unrestricted use, distribution, and reproduction in any medium, provided the original work is properly cited.

\begin{abstract}
This paper proposes a new hybrid optimization method for the phase-frequency characteristics of the double input power system stabilizer (PSS) based on the improved active set algorithm. This method takes the effect of the filtering section optimization on the parameter improvement into account, and the optimized model focuses on the minimum residual sum of squares between the actual and the target phase-frequency characteristics. The result shows that the improved parameters obtained from the proposed method provide much better phase-frequency characteristics than the widely used engineering parameters. The comparison between the proposed method and the typical commercial software indicates the universal superiority of the proposed method. And the studies on the impact of considering the filter section optimization on the phase-frequency improvement show that taking the filter section optimization into account will be beneficial for the phase-frequency improvement, though in application to the PSS2A model and the PSS2B model there are some differences. The achievements obtained in this paper provide a significant reference for the practical PSS parameter modification and improvement.
\end{abstract}

\section{Introduction}

With the fast development of the ultrahigh voltage grid, the high-capacity power transmission among different districts becomes more and more popular, resulting in the significant change of the low-frequency oscillation mode of the regional power grids $[1,2]$. Generally, two kinds of low-frequency oscillation modes at the range of $0.1 \mathrm{~Hz}-2 \mathrm{~Hz}$, that is, the partial mode and the regional mode, will be caused due to the low damping [3].

In recent years, the enlargement of the power grid has gradually reduced the natural frequency of the system and meanwhile caused the low-frequency oscillation accident to be more serious [4]. Correspondingly, most of the generators whose capacities are more than $300 \mathrm{MW}$ in China have installed a PSS. However, many parameters need to be modified accordingly to make PSS perform more efficiently and robustly. How to optimize the PSS parameters is the significant problem to provide the system with the best damping and the partial and regional grids with the better reliability and strength.
In the multigenerator system, there are generally two kinds of PSS parameter design methods [5]. The first kind is to calculate the PSS parameters according to the predefined indexes such as the damping ration and the pole locations, specifically aiming at a certain performing condition. This kind of method is advantaged in the strict theory and the accurate calculation, while actually it is weak in the robustness and the convergence [6]. The other kind is to make the PSS parameters satisfy the wide variety of the performing conditions. This kind of method, which is generally based on the phase compensation and focuses on the robustness, has been widely used [7]. By far, most of the practical PSS types are of PSS2A and PSS2B, for which scholars have developed different methods [5-13] to improve their properties. Typically, for the phase-frequency characteristics compensation, researchers have proposed various optimization studies [5, $6,8,9]$ and have founded a fine basis for the practical application.

However, most of the proposed work only focused on the phase shifters, while the practicability has not yet been 


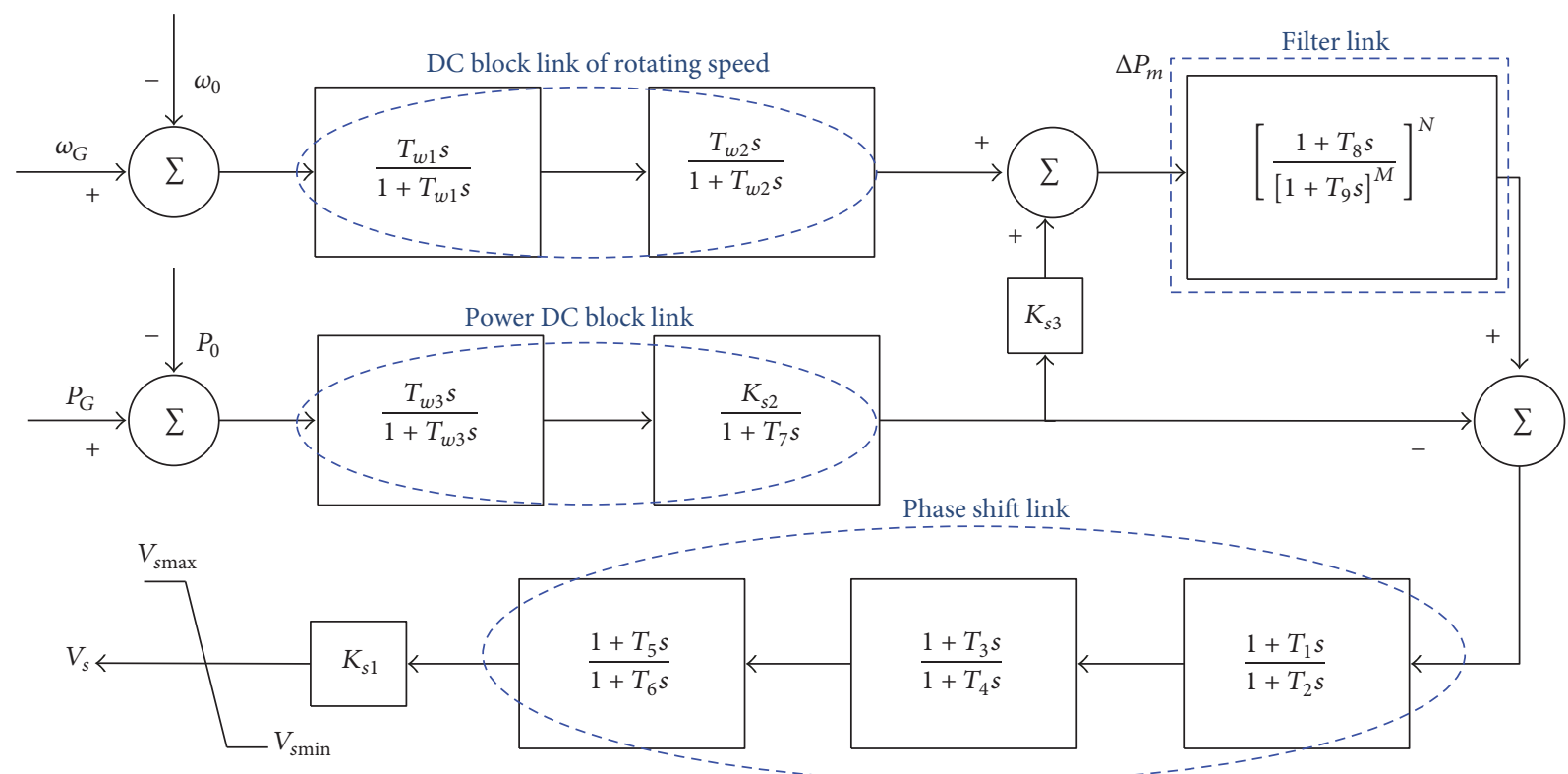

FIGURE 1: The block diagram of double input PSS2B model.

well verified. Although scholars have also developed a new improved PSS model with the double-signal input to optimize the low-frequency and high-frequency features $[7,10]$, the new model is still in the theory demonstration period and relatively lacks implement basis. To make an improvement, researchers presented parameter optimization methods for PSS4B and have verified the effectiveness by simulations and experiments [11-13]. However, since PSS4B is based on a parallel connected mode and is much more complex $[7,13]$, it has not yet been widely used in the practical engineering.

This paper proposes a new hybrid optimization method for the phase-frequency characteristic improvement based on Active Set Algorithm and Chaos Algorithm. This method not only improves the phase shifters, but also optimizes the DC blockers. The application effect comparison between the proposed method and the commonly used commercial software both for PSS2A and for PSS2B verifies the effectiveness and the advantages of the method.

\section{Engineering Phase-Frequency Characteristic Improvement of Double Input PSS}

2.1. Double Input PSS. Due to the suppression effect on the inverse reactive adjustment based on the compound of the power input branch and the rotating speed input branch [5], the double input PSS, especially the PSS2A type, has been widely used in the power systems. Typically, the PSS2B model is illustrated in Figure 1, where the rotating-speed DC blocker and the power DC blocker are used to filter the DC components, the filter section is used to filter the noises and the torsional oscillation signals in the frequency-deviation series, and the phase shifter is used to modify the frequency characteristics. When $T_{5}$ equals $T_{6}$, the third phase shifter will be invalid. In this case, the PSS2B model is equivalent to the PSS2A model which has only two phase shifters.
Currently, the technique standard [14] primarily considers the phase-frequency characteristic improvement, while ignoring the amplitude-frequency characteristic optimization. It is mainly because the amplitude-frequency characteristic will be affected little by the parameter variation but mostly influenced by the model structures (the practical PSS has a certain model structure) $[7,10]$. Limited by the unit security request and the calculation complexity constraint, no matter in the theory study $[11,12]$ or the practical application $[5,6,8,15]$, most optimization methods primarily focus on the parameter improvement of the phase shifters, while specific attention has been rarely paid to the DC blockers.

Objectively, both the system oscillation and the active mechanical torsion adjustment will cause power variations to the generator. And the power variation will further make the PSS modify the reactive power output of the generator through the exciting system. However, the reactive power modification caused by the active mechanical torsion adjustment, which is usually called reactive power inversemodification problem, will be harmful to the generator. To solve this very problem, the composite additive signal of the rotating speed and the power, namely, $\Delta P_{m}$, is employed as the input of the torsional filter link. Its basic scheme is to make the additive signal $\Delta P_{m}$ indicated in Figure 1 near zero, so that the reactive power inverse-modification effect can be mostly restrained. Therefore, theoretically, the parameter modification of the filter section will not affect the damping effect of the whole PSS [16]. This means the filter section can be ignored during the analysis on the key transfer function of the system. Only taking the power input branch into account, the transfer function of PSS can be written as

$$
\begin{aligned}
G(s)= & K_{s 1} \frac{T_{w 3} s}{1+T_{w 3} s} \cdot \frac{K_{s 2}}{1+T_{7} s} \cdot \frac{1+T_{1} s}{1+T_{2} s} \cdot \frac{1+T_{3} s}{1+T_{4} s} \\
& \cdot \frac{1+T_{5} s}{1+T_{6} s}
\end{aligned}
$$


TABLE 1: Parameters for engineering application in Dingzhou power plant.

\begin{tabular}{lcccccccc}
\hline Parameters & $T_{1}$ & $T_{2}$ & $T_{3}$ & $T_{4}$ & $T_{5}$ & $T_{6}$ & $T_{W 3}$ & $T_{7}$ \\
Values & 0.2 & 0.03 & 0.2 & 0.02 & 0 & 0 & 5 & 5 \\
\hline
\end{tabular}

and the phase-frequency expression of the PSS2B model based on (1) is

$$
\begin{aligned}
\phi_{p}= & \frac{\pi}{2}-\arctan \left(\omega T_{w 3}\right)-\arctan \left(\omega T_{7}\right) \\
& +\arctan \left(\omega T_{1}\right)-\arctan \left(\omega T_{2}\right)+\arctan \left(\omega T_{3}\right) \\
& -\arctan \left(\omega T_{4}\right)+\arctan \left(\omega T_{5}\right)-\arctan \left(\omega T_{6}\right) .
\end{aligned}
$$

Setting $T_{5}$ equivalent to $T_{6}$, the phase-frequency characteristic formula of the PSS2A model can be obtained:

$$
\begin{aligned}
\phi_{p}= & \frac{\pi}{2}-\arctan \left(\omega T_{w 3}\right)-\arctan \left(\omega T_{7}\right) \\
& +\arctan \left(\omega T_{1}\right)-\arctan \left(\omega T_{2}\right)+\arctan \left(\omega T_{3}\right) \\
& -\arctan \left(\omega T_{4}\right) .
\end{aligned}
$$

When the DC blockers are not taken into optimization, the parameters $T_{w 3}$ and $T_{7}$ in (2) and (3) will be usually set as constants.

2.2. Phase-Frequency Characteristic Improvement in Engineering. The phase-frequency improvement is usually performed based on the operators' practical experiences and the commercial software. For instance, the practical engineering method is to use engineers' professional experiences to set the PSS parameters in the commercial software firstly and then verify the parameters according to the technical standards required in [14].

Taking 1\# generator unit in Dingzhou power plant, Hebei Province, China, as an example, the detailed parameters are shown in Table 1 [15]. It can be found from Table 1 that the third-phase shifter is ignored and the model is actually a PSS2A type. The phase-frequency characteristic curves with compensation and without compensation, respectively, are indicated in Figure 2.

\section{PSS Phase-Frequency Optimization Based on Improved Active Set Algorithm}

3.1. Optimization Target and Restraint. The relation between the input signal and the phase compensation is indicated in Figure 3, where $M_{\mathrm{adv}}$ and $M_{\mathrm{lag}}$ are the lower and the upper thresholds of $M$, respectively. The phase compensation is to make the angle between the damper torque $M$ and the rotating difference $\Delta \omega$ zero by modifying the PSS parameters. When the frequency lies in $[0.2,2], M_{\mathrm{adv}}$ is usually set as $20^{\circ}$ and $M_{\text {lag }}$ is set as $-45^{\circ}$. However, when the frequency lies in $[0,0.2), M_{\text {adv }}$ is set as $40^{\circ}$ and $M_{\text {lag }}$ is still set as $-45^{\circ}$ [14]. Usually, $-\Delta P$ is taken as the reference of the delay angle. Then the range between $M_{\mathrm{adv}}$ and $M_{\mathrm{lag}}$ is transferred to $\left[-70^{\circ},-135^{\circ}\right.$.

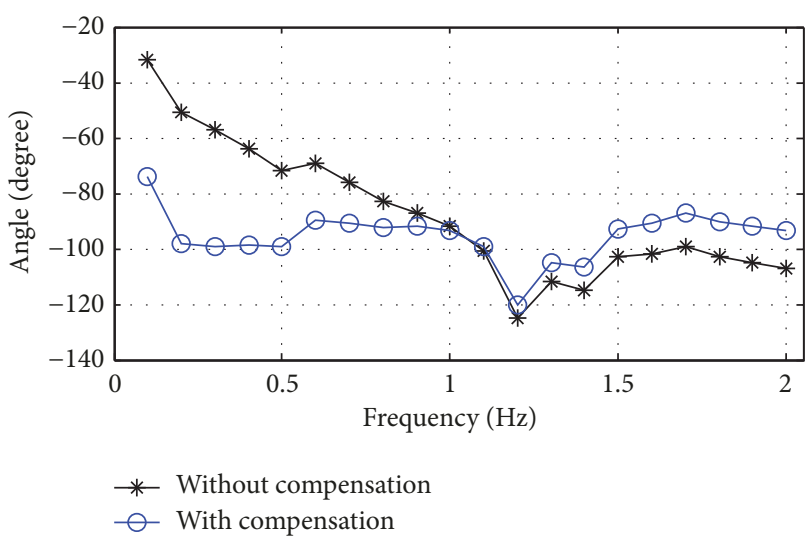

FIGURE 2: Phase-frequency characteristic of the PSS set by practical tuning method.

The ideal phase characteristic is to be consistent with $\Delta \omega$. This means the purpose of phase-frequency optimization is to set the phase angle at each frequency point to be $-90^{\circ}$ via properly modifying the PSS parameters. Commonly, the minimum residual sum of squares between the actual and the target phase-frequency curves is taken as the optimization objective which can be expressed as

$$
\min \left(\sum_{j=1}^{m}\left(\varphi_{0}\left(f_{j}\right)+\varphi_{p}\left(f_{j}\right)+90\right)^{2}\right),
$$

where $m$ is the number of the sampling frequency points from 0 to $2 \mathrm{~Hz}$. In engineering, there are usually 20 frequency points, with the intervals of $0.1 \mathrm{~Hz} . \varphi_{p}\left(f_{j}\right)$ stands for the compensation phase angle produced by PSS at $f_{j}$, which depends on the detailed PSS parameters and can be written as (3) for the PSS2A model, while $\varphi_{0}\left(f_{j}\right)$ stands for the delayed phase angle of the generator set without compensation at $f_{j}$. Technically, to test $\varphi_{0}\left(f_{j}\right)$, the periodic frequency-modification signal which includes the sine waves from 0 to $2 \mathrm{~Hz}$ is set as the input of the automatic voltage regulator (instead of the PSS signal). Then the magnitude of the input signal will be amplified until the output voltage of the generator has a slight fluctuation. Finally, the phase angle between the input signal and the output voltage of the generator is detected. This angle is the delayed angle of the generator. More details can be found in the Chinese national standard; see [14].

According to [17], only the phase shifter is taken as the optimization object, and the parameters of $T_{W_{3}}$ and $T_{7}$ are set as Table 1 , while the parameters of $T_{1} \sim T_{4}$ are set within the range of $[0.01,1]$.

3.2. Improved Active Set Algorithm. The solution of the nonlinear parametric optimization problem should consider both the efficiency and the initial values. Unreasonable initial values often lead to the local poles and the loss of the global optimal solution. Since the chaotic system has the characteristics of randomness and ergodic regularity, it can travel more evenly in the range of the variables without a repetition. In addition, it can effectively avoid the local 


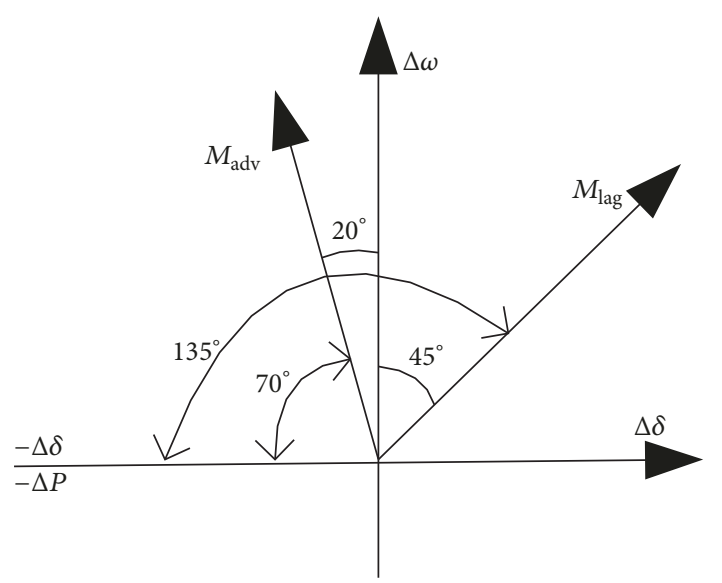

FIGURE 3: Diagram between input signal and phase compensation.

optimal values of the calculated parameters. However, chaos algorithm will be unsatisfactory when the search space is large [18]. The Active Set Algorithm, which is an effective tool to solve the boundary restricted problems $[19,20]$, is combined with the chaos algorithm in this paper to solve the boundary optimization problem shown in

$$
\begin{array}{ll}
\min & f(x) \\
\text { s.t. } & l \leq x \leq u .
\end{array}
$$

3.2.1. Initial Value Calculation Based on Chaos Algorithm. Typically, the classical logistic map which indicates the onedimensional chaotic system can be written as

$$
x_{n+1}=\mu x_{n}\left(1-x_{n}\right) \text {, }
$$

where the control parameter $\mu=4,0 \leq x_{i} \leq 1$, and $n=$ $0,1,2,3, \ldots$.

Obviously, the system indicated in (6) is in the state of chaos and sensitive to the initial values. By giving $i$ initial values, the system can be assigned with $i$ subtle different chaotic variables. Introducing the chaotic variables into the optimization, the chaotic motion range will be expanded to the scope of the optimized variable ranges. The specific steps are as follows.

Step 1. Respectively, assign $i$ subtle different initial values to $x_{i}$, and obtain $i$ different chaotic variables $x_{i, n+1}$.

Step 2. The ranges of chaotic variables are, respectively, mapped to the optimized variable ranges by

$$
x_{i, n+1}^{\prime}=c_{i}+d_{i} x_{i, n+1},
$$

where $c_{i}$ and $d_{i}$ are the constant mobile coefficients and the amplification coefficients, respectively.

Step 3. Calculate $f\left(x_{i, n+1}^{\prime}\right)$ based on the iteration search of the chaotic variables and meanwhile make a record for both the parameters and $f\left(x_{i, n+1}^{\prime}\right)$.
TABLE 2: Parameter optimization results of two phase shifters.

\begin{tabular}{lcccc}
\hline Parameters & $T_{1}$ & $T_{2}$ & $T_{3}$ & $T_{4}$ \\
Optimization results & 0.22 & 0.03 & 0.23 & 0.02 \\
\hline
\end{tabular}

Step 4. If the optimal performance indicators remain constant after several iterations as Step 3, then set the values as the initial solutions.

3.2.2. Solutions by Active Set Algorithm. In the boundary restricted problem shown in (5), $f(x)$ is a real-number function, $x \in R^{n}$ is the $n$-order real value vector, and $l$ and $u$ are the boundary vectors, respectively. Since the upper and the lower boundaries of the parameters are also the restraint conditions of (5), this equation is a boundary restricted optimization problem. The active set at the point $x^{*}$ is defined as

$$
I\left(x^{*}\right)=L\left(x^{*}\right) \cup U\left(x^{*}\right),
$$

where $L\left(x^{*}\right)=\left\{i: x_{i}^{*}=l_{i}\right\}$ and $U\left(x^{*}\right)=\left\{i: x_{i}^{*}=u_{i}\right\}$.

Assuming that (5) is the active set of the optimized answers, the problem can be transferred to an equality constraint optimization question, so that the solving difficulty is greatly reduced. However, since the optimal solving result $x^{*}$ is unknown, the active set $I\left(x^{*}\right)$ is also unknown and requires an estimation performance.

The key thought of the active set algorithm is to begin with the initial value $x^{0}$ and keep generating an estimated active set $A\left(x^{p}\right)$ for each iteration until obtaining an optimized solving result. Therefore, the key point to solve the problem is to select a proper active set strategy [21]. In this paper, we employ the approximate active set strategy proposed by Ni and Yuan [22] to solve the problem. The approximate active set $A$ and the inactive set $B$ can be written as

$$
\begin{aligned}
& A(x)=\left\{i: l_{i} \leq x_{i} \leq l_{i}+\varepsilon_{b}\right\} \cup\left\{i: u_{i}-\varepsilon_{b} \leq x_{i} \leq u_{i}\right\} \\
& B(x)=\{1, \ldots, n\} \backslash A(x)=\left\{i: l_{i}+\varepsilon_{b} \leq x_{i} \leq u_{i}-\varepsilon_{b}\right\},
\end{aligned}
$$

where $0<\varepsilon_{b}<\min _{i}\left[\left(u_{i}-l_{i}\right) / 3\right]$. During the calculation, the algorithm divides the search directions into three ones, that is, the quasi-Newton direction in the subspace expanded with the invalid parameters, the gradient direction, and the modified gradient direction in the subspace expanded with the valid parameters. The invalid parameters are updated by the limited internal storage quasi-Newton method, while the valid parameters are updated by the projection gradient method.

\subsection{Optimization Result and Discussion of Two Phase Shifters.} Taking the modified parameters shown in Table 1 as the initial values of PSS, the optimized results for the phasefrequency characteristics indicated in (4) based on the Active Set Algorithm mentioned in Section 3.2 are shown in Table 2. And the phase-frequency characteristic curves of PSS after optimization of the two phase shifters are indicated in Figure 4.

As indicated in Figure 4, it is obvious that the phasefrequency characteristic curve optimized with two phase 
TABLE 3: Key information of the oscillation mode.

\begin{tabular}{lcc}
\hline Method & Frequency & Damping ratio \\
\hline Without PSS & 1.33 & 0.0576 \\
PSS performed with the practical engineering method & 1.39 & 0.2167 \\
PSS performed with the two-phase-shifter optimized method & 1.35 & 0.2665 \\
\hline
\end{tabular}

TABLE 4: Robustness verification under different disturbance degrees.

\begin{tabular}{lccc}
\hline Step amplitude variation percentage of the exciting voltage & $22 \%$ & $44 \%$ & $66 \%$ \\
Damping ratio obtained from commercial software & 0.1705 & 0.1687 & 0.1659 \\
Damping ratio obtained from this paper & 0.2336 & 0.2285 & 0.2218 \\
\hline
\end{tabular}

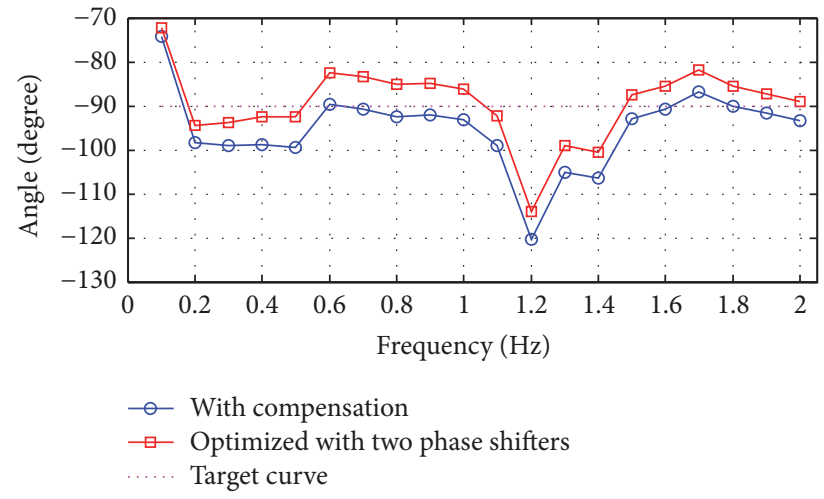

FIgURE 4: Phase-frequency characteristic curves before and after optimizations of the two phase shifters.

shifters is closer to the target curve. The sampling points beyond $\left[-80^{\circ},-100^{\circ}\right]$ are reduced from 4 to 2 , and the max phase angle deviation at $1.2 \mathrm{~Hz}$ is also changed from $-120.4^{\circ}$ to $-114^{\circ}$.

Taking the least squares calculation value between the phase-frequency characteristic curve and the target curve as the evaluation index (the smaller, the better), the index value after optimization is 1433 , which is reduced by $32.5 \%$ compared to the index value of 2122 before optimization.

3.4. Simulating Verification of Optimized Result. To further verify the practical effect, the actual offline data is employed for the simulating calculation. The result is obtained through the Prony analysis method by means of loading a step excitation and a transient fault disturbance. Keeping the gain coefficient $K_{s 1}$ (see Figure 1) stable in different cases, the responses are illustrated in Figure 5, and the key information about the oscillation mode is shown in Table 3 .

It is suggested from Figure 5 that, by loading the same disturbance, PSS has an obvious inhibiting effect on the power oscillation. Without PSS, it needs more than 5 cycles to quiet down the oscillation, while with PSS it needs only 3 oscillation cycles. Employing the PSS of which the two phase shifters are optimized by the proposed method in this paper, it only needs two cycles to remove the oscillation. Moreover, the oscillation magnitudes are apparently smaller. As indicated in Table 3, the damping ratio after optimization is 0.2665 , which is

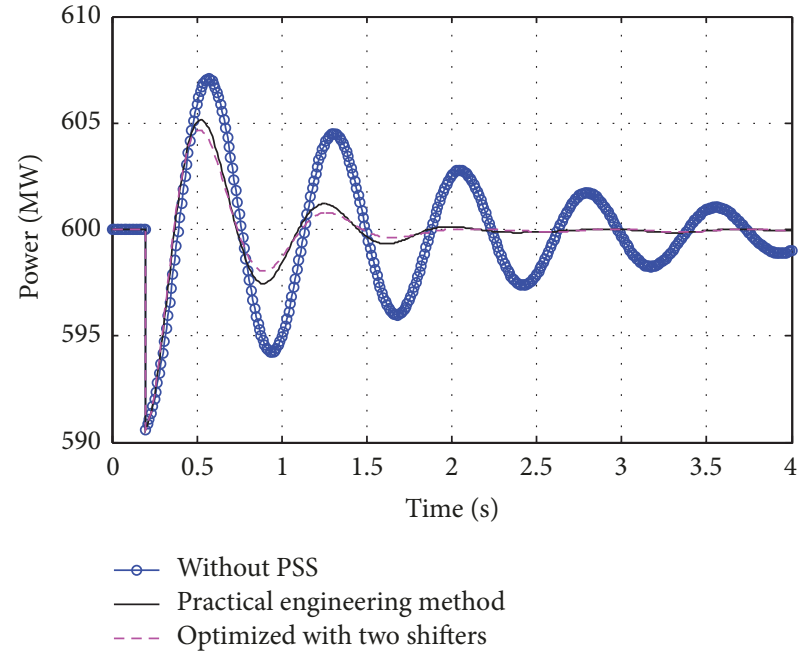

Figure 5: Power oscillation responses for a transient disturbance.

increased by $23 \%$ compared to the practical engineering method.

In addition to the effectiveness of the optimization method, the robustness is also a key point. To verify the robustness of the proposed method, a comparison between the proposed method and the commercial software (PSS Parameter Calculation Program developed by China Electric Power Research Institute) is taken. The step amplitude variation percentage of the exciting voltage is used as a disturbance, and the damping ratios obtained from the commercial software and the proposed method are indicated in Table 4. It is shown that the proposed method has a better damping ratio, suggesting the better robustness of the proposed method.

\section{Effect Comparisons with Commercial Software}

Employing the commercial software PSS Parameter Calculation Program which is widely used in China and the proposed method in this paper, respectively, to calculate the optimization parameters of 1 \# generator set in Dingzhou Power Plant, the results are shown in Table 5, and the phase-frequency 
TABLE 5: Optimized parameter obtained by commercial software.

\begin{tabular}{lcccc}
\hline Parameter & $T_{1}$ & $T_{2}$ & $T_{3}$ & $T_{4}$ \\
Optimized value & 0.16 & 0.02 & 0.3 & 0.03 \\
\hline
\end{tabular}

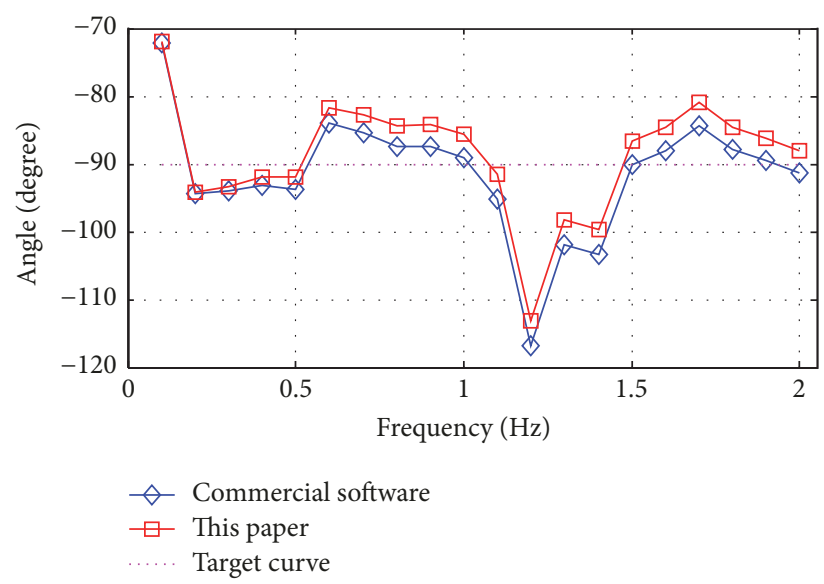

FIGURE 6: Phase-frequency characteristic curves obtained by the commercial software and the proposed method.

characteristic curves are indicated in Figure 6. The index value for the commercial software is 1556 (the smaller the better), while the index value of the proposed method is 1433 . It is shown that the proposed method is $8 \%$ superior to the widely used commercial software.

Objectively, we also employ another 4 actual generator sets of PSS2A type to make a further verification. The phasefrequency characteristic curves are indicated in Figure 7, while the optimized parameters and the evaluation index values are indicated in Table 6.

As indicated in Figure 7, the phase-frequency characteristic curves optimized by the proposed method are closer to the target curve than those obtained from the commercial software. The evaluation index values for the 4 optimized generator sets are reduced by $37.9 \%, 27.6 \%, 22.3 \%$, and $24.9 \%$, respectively, showing that the proposed method is universal and has a generally better optimized effect.

\section{Expanded Optimization of Phase-Frequency Characteristics}

5.1. Parameter Optimization in Two Phase Shifters and DC Blockers. The primary difference between the PSS2A model and the PSS2B model is the number of the phase shifters. The PSS2B model has three phase shifters, while the PSS2A model has only two phase shifters (see Section 2.1).

For the PSS2A model, the initial values of the PSS parameters are set as Table 1 to study the improved effect of optimizing the DC blockers and the two phase shifters at the same time. The improved phase-frequency characteristics are calculated by the method mentioned in Section 3.2 based on (4). The detailed expression of $\varphi_{p}\left(f_{j}\right)$ is indicated as (3), where $T_{1} \sim T_{4}, T_{W 3}$, and $T_{7}$ are the optimization parameters. According to [17], the values of $T_{W 3}$ and $T_{7}$ are in the range of $[4,6]$, while the values of $T_{1} \sim T_{4}$ are in the range of $[0.01,1]$.
The optimized parameters obtained from the proposed method are shown in Table 7 , and the optimized phasefrequency curves obtained from the proposed method and the practical engineering method, respectively, are indicated in Figure 8.

According to Table 7, the evaluation index value of the optimized parameters is 1313 , which is $38.1 \%$ superior to the practical engineering method. Comparing with the twophase-shifter optimization case, the expanded optimization method improves the effect by $8.4 \%$. As illustrated in Figure 8 , although the improved extent is not very large, the proposed hybrid optimization method is still worth to be carried out to furthest obtain the best phase-frequency characteristics.

\subsection{Parameter Optimization in Three Phase Shifters and DC} Blockers. For the PSS2B model, the optimization work acts on the DC blockers and the three phase shifters at the same time. The initial values of the PSS parameters are set as Table 1, and the improved phase-frequency characteristics are calculated by the method mentioned in Section 3.2 based on (4). The detailed expression of $\varphi_{p}\left(f_{j}\right)$ is as (2). $T_{1} \sim T_{6}, T_{W 3}$, and $T_{7}$ are the optimization parameters. According to [17], the value of $T_{W 3}$ and $T_{7}$ is in the range of $[4,6]$, while the value of $T_{1} \sim T_{6}$ is in the range of $[0.01,1]$.

The improved parameters obtained by the proposed hybrid optimization method are shown in Table 8 , and the optimized phase-frequency curves obtained by the proposed method and the practical engineering method are indicated in Figure 9.

According to Table 8, the evaluation index value of the hybrid optimized parameters is 1243 , which is $41.4 \%$ superior to the practical engineering method and 5.3\% superior to the two-phase-shifter optimization case. Moreover, the hybrid optimization method has the best improving effect both at the frequency point $0.1 \mathrm{~Hz}$ and at the frequency band from 1.2 to $1.4 \mathrm{~Hz}$ (these frequency points have been paid much attention by scholars). This means the hybrid optimization method has a better effect on the phase-frequency characteristic improvement at the extreme points.

Since the PSS2A model is less complex than PSS2B and has fewer parameters to modify, the PSS2A model which has qualified accuracy is more widely used in the engineering application. The PSS2B model is usually only used for the generators whose phase-frequency characteristics are obviously weak.

\section{Conclusions}

This paper proposes a new hybrid optimization method for the double input PSS. The comparing results show that this method is able to significantly improve the phase-frequency characteristics for both the PSS2A model and the PSS2B model. The primary conclusions drawn from the study are as follows.

(1) The practical engineering method and the commonly used commercial method only take the phase shifter optimization into account, while ignoring the DC blocker improvement. Actually, it is better to optimize the phase shifters and the DC blockers at the same time. 
TABLE 6: Optimized parameters and evaluation index values obtained by the commercial software and the proposed method.

\begin{tabular}{|c|c|c|c|c|c|c|}
\hline \multicolumn{2}{|c|}{ Parameters } & $T_{1}$ & $T_{2}$ & $T_{3}$ & $T_{4}$ & Index value \\
\hline \multirow{2}{*}{ Generator set 1} & Commercial software & 0.30 & 0.03 & 0.30 & 0.03 & 3389 \\
\hline & This paper & 0.13 & 0.01 & 0.33 & 0.01 & 2103 \\
\hline \multirow{2}{*}{ Generator set 2} & Commercial software & 0.15 & 0.02 & 0.30 & 0.03 & 1977 \\
\hline & This paper & 0.24 & 0.01 & 0.12 & 0.01 & 1432 \\
\hline \multirow{2}{*}{ Generator set 3} & Commercial software & 0.19 & 0.02 & 0.2 & 0.03 & 1768 \\
\hline & This paper & 0.17 & 0.01 & 0.16 & 0.01 & 1373 \\
\hline \multirow{2}{*}{ Generator set 4} & Commercial software & 0.19 & 0.02 & 0.2 & 0.03 & 5677 \\
\hline & This paper & 0.17 & 0.01 & 0.16 & 0.01 & 4261 \\
\hline
\end{tabular}
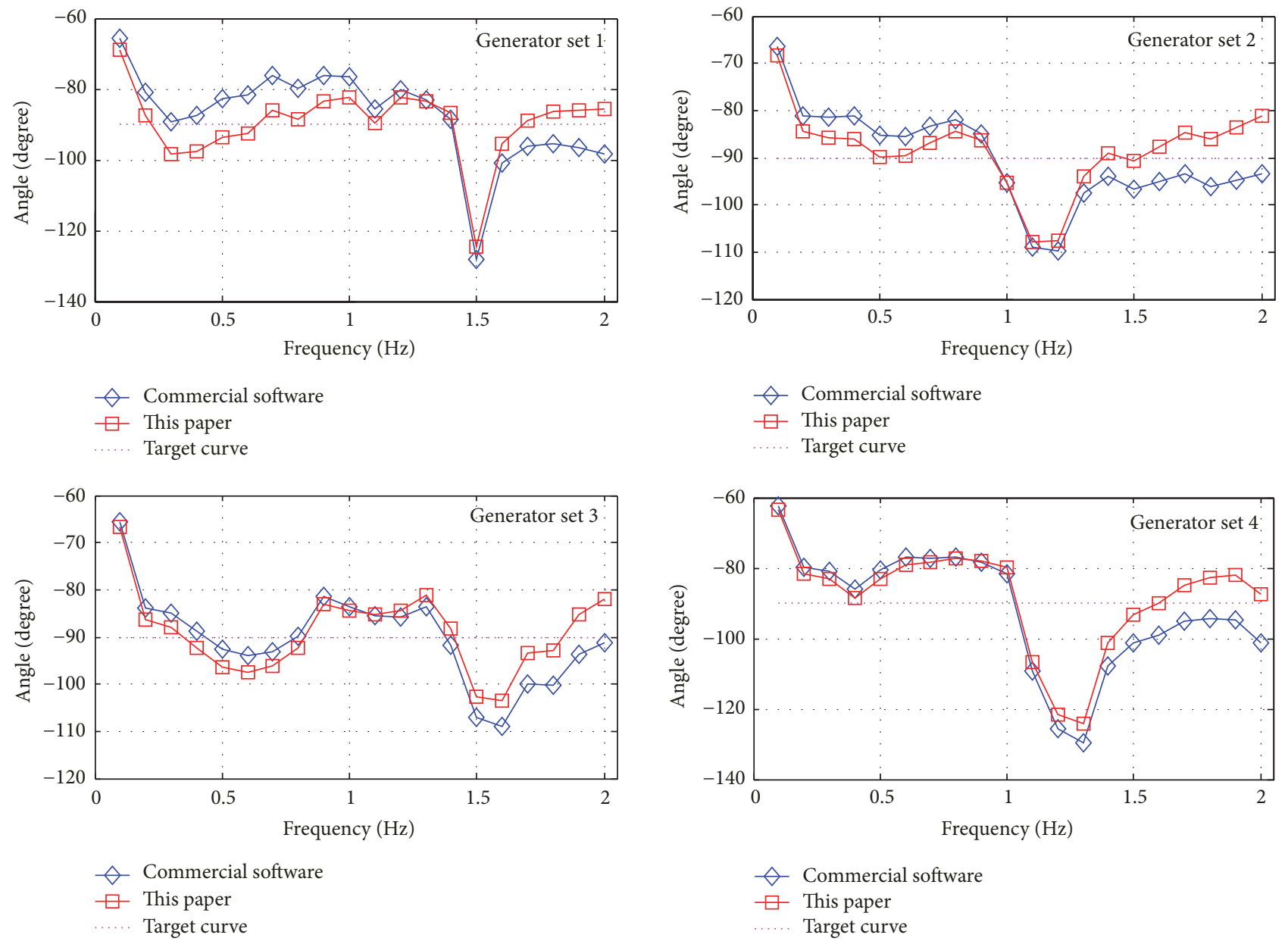

FIgURE 7: Phase-frequency characteristic comparison between the commercial software and the proposed method.

TABLE 7: Optimized parameters of the DC blockers and the two phase shifters.

\begin{tabular}{lcccccc}
\hline Parameters & $T_{1}$ & $T_{2}$ & $T_{3}$ & $T_{4}$ & $T_{W 3}$ & $T_{7}$ \\
Optimized value & 0.23 & 0.02 & 0.23 & 0.03 & 6 & 6 \\
\hline
\end{tabular}

(2) This paper proposes a new hybrid optimization method based on Chaos Algorithm and Active Set Algorithm to improve the PSS parameters. The Prony simulating calculation and the actual offline data verification have confirmed
TABLE 8: Optimized parameters of the DC blockers and the three phase shifters.

\begin{tabular}{lcccccccc}
\hline Parameters & $T_{1}$ & $T_{2}$ & $T_{3}$ & $T_{4}$ & $T_{5}$ & $T_{6}$ & $T_{W 3}$ & $T_{7}$ \\
Optimized results & 0.18 & 0.05 & 0.19 & 0.05 & 0.18 & 0.05 & 6 & 6
\end{tabular}

the effectiveness of the proposed method. Moreover, this algorithm does not need manually specified initial values.

(3) The comparing result shows that the optimized effect of the proposed method is superior to the practical engineering method and the commercial software. 

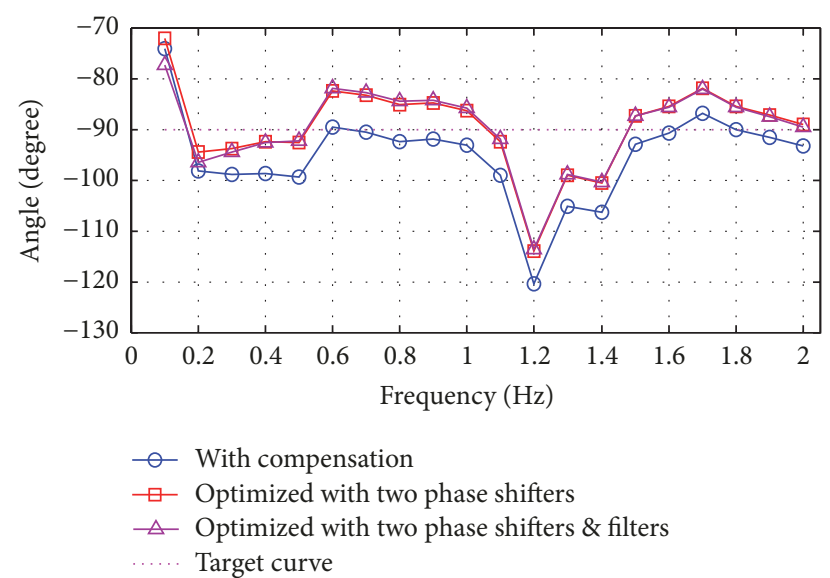

FIGURE 8: Optimized phase-frequency characteristic curves.

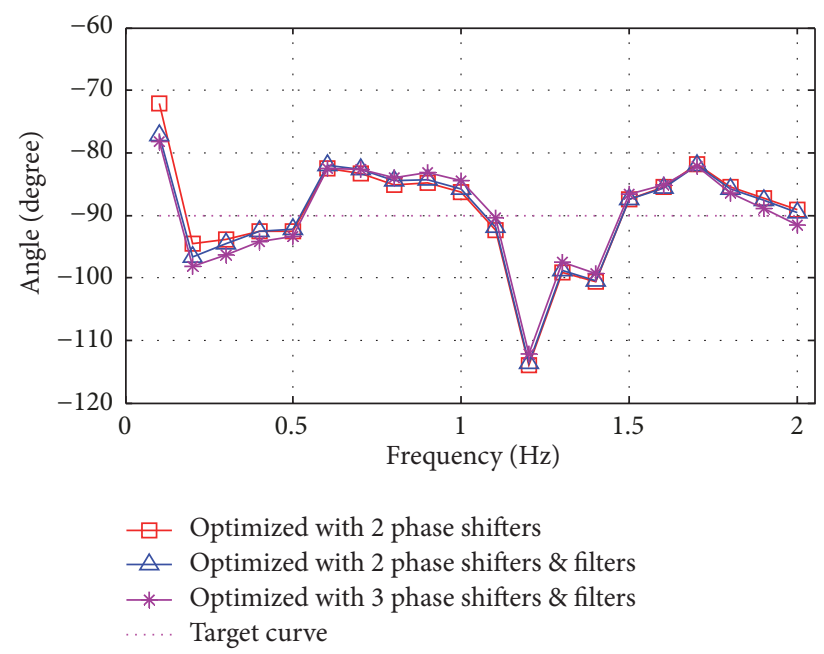

FIGURE 9: Optimized phase-frequency characteristics obtained by different methods.

\section{Conflicts of Interest}

The authors declare that they have no conflicts of interest.

\section{Acknowledgments}

This work is in part supported by National Natural Science Foundation of China (51307058, 51777074), Natural Science Foundation of Hebei Province, China (E2015502013), and Chinese Fundamental Research Funds for the Central Universities (2018YQ03).

\section{References}

[1] J. Zhang, C. Y. Chung, and Y. Han, "A novel modal decomposition control and its application to PSS design for damping interarea oscillations in power systems," IEEE Transactions on Power Systems, vol. 27, no. 4, pp. 2015-2025, 2012.

[2] S. Kamalasadan and G. D. Swann, "A novel system-centric intelligent adaptive control architecture for damping interarea mode oscillations in power system," IEEE Transactions on Industry Applications, vol. 47, no. 3, pp. 1487-1497, 2011.

[3] M. Xiao-Ming, Z. Yao, G. Lin, and W. Xiao-Chen, "Coordinated control of interarea oscillation in the China Southern power grid," IEEE Transactions on Power Systems, vol. 21, no. 2, pp. 845-852, 2006.

[4] J. He, C. Lu, X. Wu, P. Li, and J. Wu, "Design and experiment of wide area HVDC supplementary damping controller considering time delay in china southern power grid," IET Generation, Transmission \& Distribution, vol. 3, no. 1, pp. 17-25, 2009.

[5] J. Ma, H. J. Wang, and K. L. Lo, "Clarification on power system stabiliser design," IET Generation, Transmission and Distribution, vol. 7, no. 9, pp. 973-981, 2013.

[6] A. Kumar, "Power system stabilizers design for multimachine power systems using local measurements," IEEE Transactions on Power Systems, vol. 31, no. 3, pp. 2163-2171, 2016.

[7] W. Kuayu, L. Cencen, L. Long et al., "A new PSS with doublesignal input and its simulation research," Power System Technology, vol. 40, no. 5, pp. 1462-1468, 2016.

[8] F. S. Al-Ismail, M. A. Hassan, and M. A. Abido, "RTDS implementation of STATCOM-based power system stabilizers," Canadian Journal of Electrical and Computer Engineering, vol. 37, no. 1, Article ID 6823225, pp. 48-56, 2014.

[9] J. Zhang, C. Y. Chung, S. Zhang, and Y. Han, "Practical wide area damping controller design based on ambient signal analysis," IEEE Transactions on Power Systems, vol. 28, no. 2, pp. 16871696, 2013.

[10] S. Zhang and F. L. Luo, "An improved simple adaptive control applied to power system stabilizer," IEEE Transactions on Power Electronics, vol. 24, no. 2, pp. 369-375, 2009.

[11] D. Rimorov, I. Kamwa, and G. Joós, "Model-based tuning approach for multi-band power system stabilisers PSS4B using an improved modal performance index," IET Generation, Transmission \& Distribution, vol. 9, no. 15, pp. 2135-2143, 2015.

[12] A. Yaghooti, M. Oloomi Buygi, and M. H. M. Shanechi, "Designing coordinated power system stabilizers: a reference model based controller design," IEEE Transactions on Power Systems, vol. 31, no. 4, pp. 2914-2924, 2016.

[13] I. Kamwa, R. Grondin, and G. Trudel, "IEEE PSS2B versus PSS4B: the limits of performance of modern power system stabilizers," IEEE Transactions on Power Systems, vol. 20, no. 2, pp. 903-915, 2005.

[14] National Energy Bureau, DL/T 1231-2013 Experiment Principles of PSS Setting, China Electric Power Press, Beijing, China, 2013.

[15] Y. Chang, PSS Experiment of 1\# Generator Set in Guohua Dingzhou Power Generation Co., Ltd, Report in State Grid Hebei Electric Power Research Institute, Shijiazhuang, China, 2015.

[16] M. A. Masrob, M. A. Rahman, and G. H. George, "Design of a neural network based power system stabilizer in reduced order power system," in Proceedings of the 30th IEEE Canadian Conference on Electrical and Computer Engineering, CCECE '17, pp. 1-6, 2017.

[17] Y. Liu, Q. H. Wu, H. Kang, and X. Zhou, "Switching power system stabilizer and its coordination for enhancement of multi-machine power system stability," CSEE Journal of Power and Energy Systems, vol. 2, no. 2, pp. 98-106, 2016.

[18] C.-H. Huang, C.-H. Lin, and C.-L. Kuo, "Chaos synchronization-based detector for power-quality disturbances classification in a power system," IEEE Transactions on Power Delivery, vol. 26, no. 2, pp. 944-953, 2011. 
[19] Q. Fan, Y. Jiao, and X. Lu, "A primal dual active set algorithm with continuation for compressed sensing," IEEE Transactions on Signal Processing, vol. 62, no. 23, pp. 6276-6285, 2014.

[20] D. R. Musicant and A. Feinberg, "Active set support vector regression," IEEE Transactions on Neural Networks and Learning Systems, vol. 15, no. 2, pp. 268-275, 2004.

[21] H. Chung, E. Polak, and S. Sastry, "An external active-set strategy for solving optimal control problems," Institute of Electrical and Electronics Engineers Transactions on Automatic Control, vol. 54, no. 5, pp. 1129-1133, 2009.

[22] Q. Ni and Y. Yuan, "A subspace limited memory quasi-Newton algorithm for large-scale nonlinear bound constrained optimization," Mathematics of Computation, vol. 66, no. 220, pp. 1509-1520, 1997. 


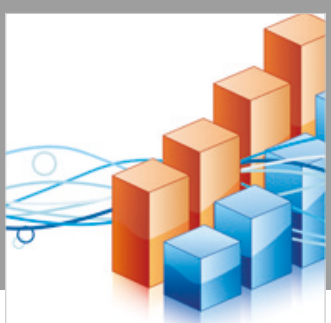

Advances in

Operations Research

\section{-n-m}
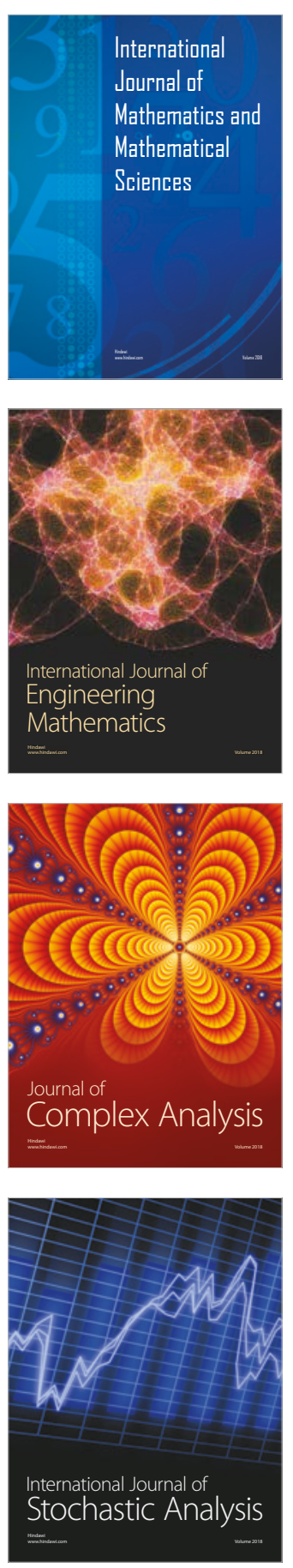
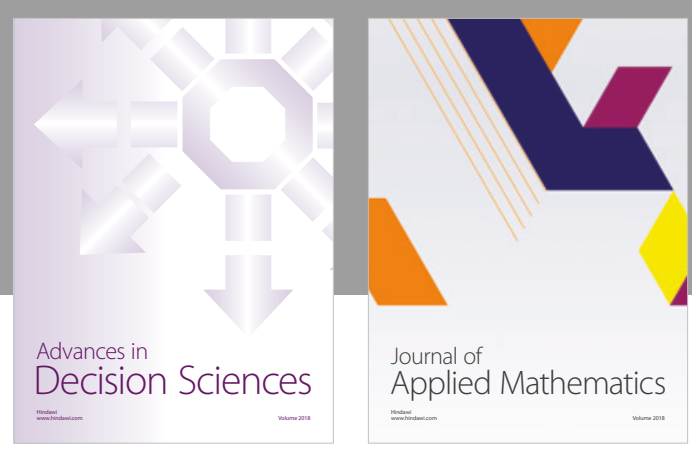

Journal of

Applied Mathematics
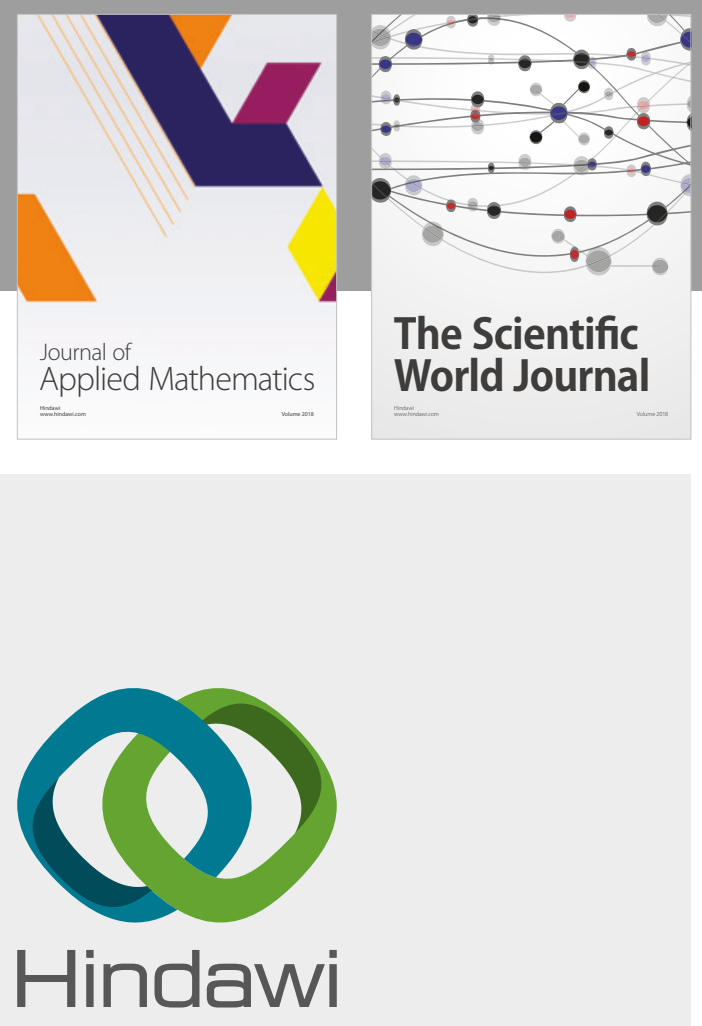

Submit your manuscripts at

www.hindawi.com

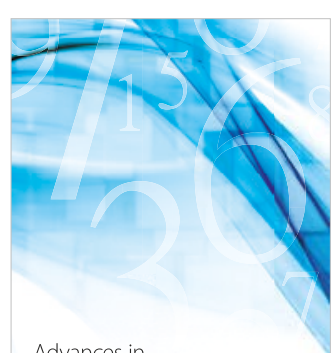

Advances in
Numerical Analysis
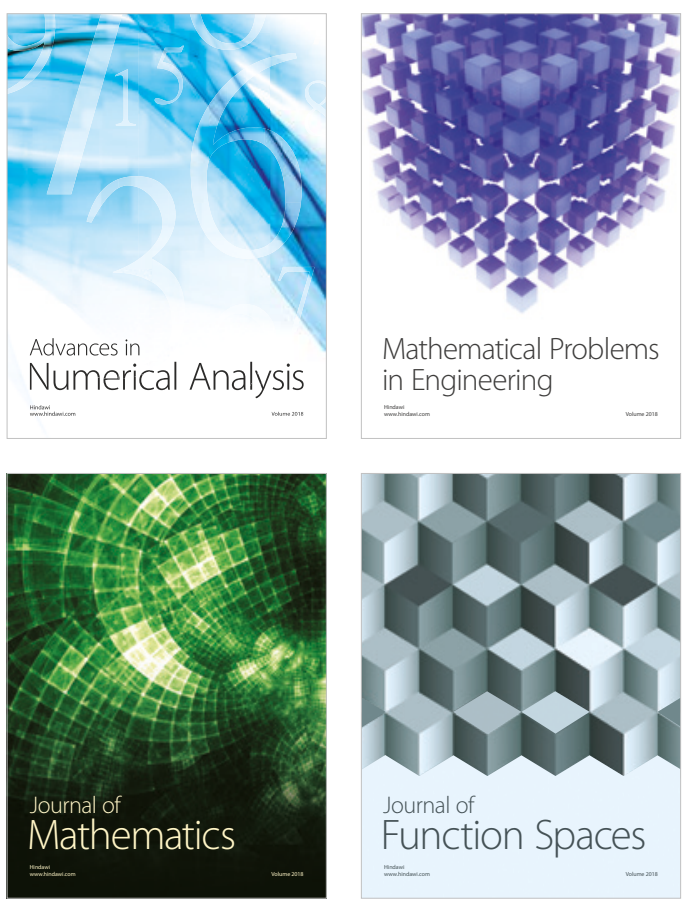

Mathematical Problems in Engineering

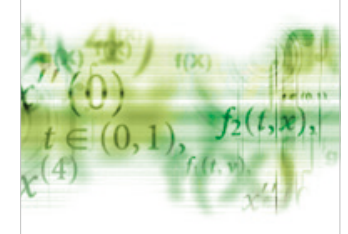

International Journal of

Differential Equations

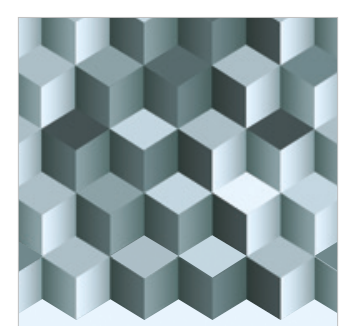

Journal of

Function Spaces

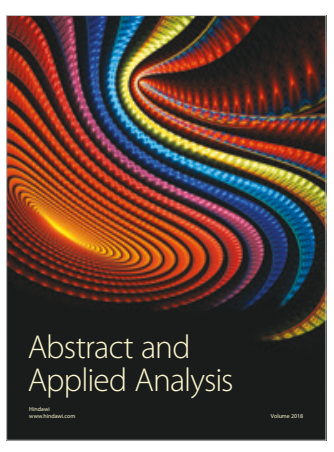

The Scientific

World Journal

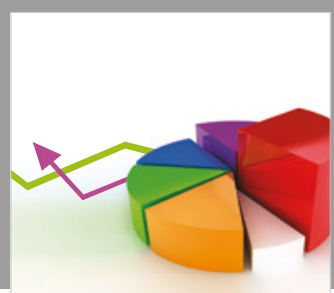

Journal of

Probability and Statistics
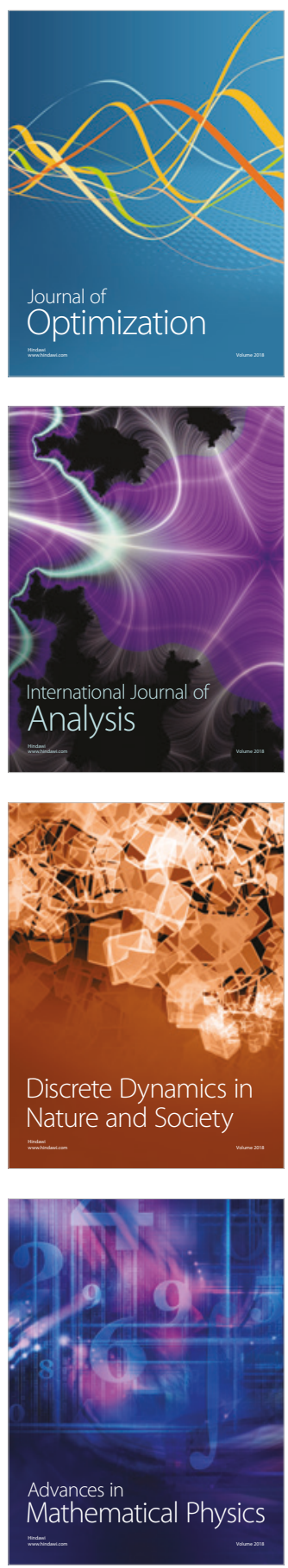